\title{
¿En dónde quedó el poder de las mujeres?
}

\author{
María Teresa Reyes Chacon
}

\begin{abstract}
Resumen
La mujer del siglo xxı persiste a la sombra del género masculino en el ámbito laboral. El discurso social, político y organizacional pregona equidad; sin embargo, en la cotidianeidad la realidad es otra: tras un análisis de publicaciones en escenarios políticos y corporativos, se detectan y describen diferencias que persisten entre los géneros al interior de las organizaciones en cualquier ámbito. En este sentido, el peso de la cultura sobre el comportamiento de las mujeres en lo laboral aún ejerce un impacto decisivo para su desarrollo corporativo. El objetivo del presente ensayo es enunciar algunos factores que prevalecen como limitantes para el ascenso de las mujeres dentro del área laboral.
\end{abstract}

Palabras clave: equidad, participación, ámbito laboral, género.

\section{WHERE IS THE POWER OF WOMEN? THE PROBLEM OF THE GENDER GAP}

\begin{abstract}
In the 21st century, women at the workplace are still at the shadow of male gender. The social, political and organizational discourse proclaims equality and/or equity; however, in everyday life the reality is other: after an analysis of publications in political and corporate settings, we describe the differences that persist between genders within organizations in any field. The weight of culture on the behavior of women at the workplace still exerts a decisive impact on their corporate development. The objective of this essay is to state some factors that prevail as limiting for the promotion of women at workplaces.
\end{abstract}

Keywords: equity, participation, work environment, gender.

Recepción: 03/03/2020. Aprobación: 04/02/2021. Dol: http://doi.org/10.22201/cuaieed.16076079e.2021.22.3.1 


\section{María Teresa Reyes Chacon}

tesareyes@gmail.com

orcid.org/0000-0003-4117-0607

Médico Familiar de 46 años de edad, nacida y educada en Chihuahua. Formación en medicina por la UACH, con experiencia en el campo clínico por más de 20 años, especialidad de médico familiar en 2008 por la UACJ. Con adscripción laboral en el Imss de Ciudad Juárez; desde el 2010 se presentó la oportunidad de desempeñar el cargo de coordinador médico por lo que realizó estudios en administración tanto maestría como doctorado en ese campo. Adicionalmente lleva a cabo estudios en educación a nivel maestría.

Ha incursionado en áreas de formación de recursos humanos para la salud, así como en áreas académicas, como ponente en congresos, foros, talleres y mesas redondas, tanto en el campo clínico como en el administrativo. Tiene un interés particular en desarrollar investigación en administración de unidades médicas y en el área clínica epidemiológica.

\section{Introducción}

Las mujeres, desde la década de los años 50, han salido a trabajary han participado en las actividades económicas de la sociedad. Así, se ha incrementado el número de mujeres en campos laborales, académicos o culturales, con cargos de poder y en la toma de decisiones. En países como Alemania y Chile las mujeres han desempeñado cargos de alto nivel, como presidentas de la nación; en Inglaterra una mujer ha desempeñado el cargo de primera ministra en dos ocasiones; en México, por otro lado, la mujer ha incrementado el grado escolar que ostenta, ha amplificado las filas laborales y participa activamente en el desarrollo cultural. Sin embargo, no figura en cargos de alta dirección, públicos o privados.

Llama la atención que las mujeres destaquen en áreas específicas como manejo de recursos humanos, áreas sociales y culturales, con lo que persisten las distinciones entre las áreas "masculinas" y "femeninas".

Imagen 1. Las mujeres han destacado en puestos de manejo de recursos humanos. Fuente: fotografía propia.

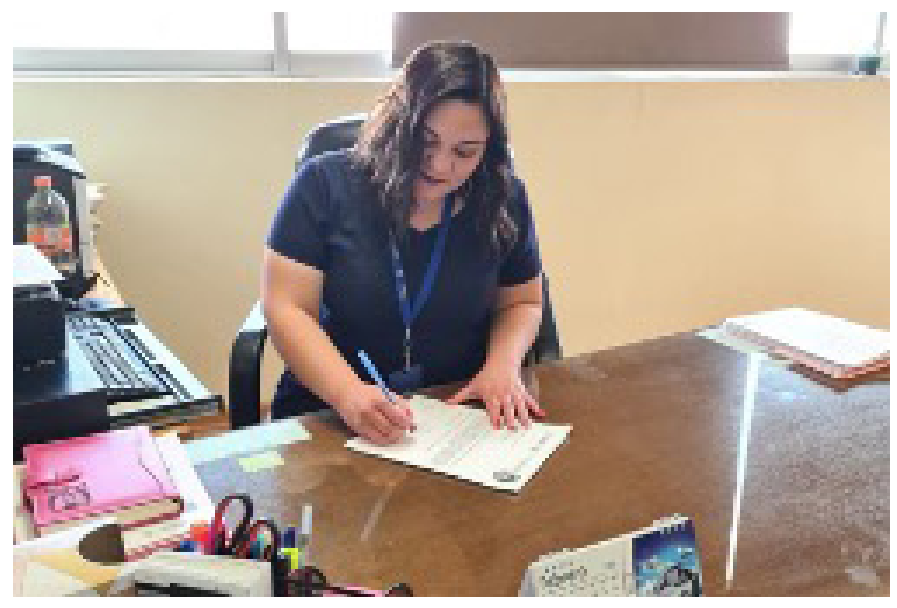


"¿En dónde quedó el poder de las mujeres?"

María Teresa Reyes Chacon

Vol. 22, Núm. 3, mayo-junio 2021

Revista Digital Universitaria

Según datos de la Asociación Nacional de Universidades e Instituciones de Educación Superior (ANUIES) del año 2013, el porcentaje de titulación para las mujeres a nivel licenciatura es de 48\%. En el nivel de posgrado, sólo 50.4\% de las mujeres obtiene el título, y en especialidad y maestría, 51\%. De esta manera, el poder de las mujeres se asevera en documentos oficiales, discursos, actas internacionales y promocionales de televisión, pero en la vida real la situación dista de ser la acordada.

Así, de acuerdo con María del Carmen Bernal y Alejandra Moreno Maya, adscritas al Centro de Investigación de la Mujer en la Alta Dirección (CIMAD) de la escuela de negocios mexicana IPADE, en México únicamente 4\% de las mujeres ocupan puestos de alta dirección o CEO ${ }^{1}$ (s.f.). Es decir, 84\% de los puestos en los consejos directivos están a cargo de hombres, y únicamente 16\% los sustentan mujeres, según el Reporte del Censo de Diversidad de Mujeres y Minorías en los Consejos Directivos, de la consultora Deloitte (Diversity y Deloitte, 2019).

El discurso político se ha manifestado abiertamente en favor del empoderamiento de la mujer, por lo que se promueven y alientan nuevas y mayores oportunidades para la participación de las mujeres en la vida social, económica y de gestión en México. Como consecuencia de tal apoyo, es de esperarse que las mujeres despunten en el campo de la política o en el ejercicio del poder dentro del Estado y que asciendan jerárquicamente dentro de la institución.

En este orden de ideas, en el 2014 un boletín emitido por el gobierno de la república y el instituto de las mujeres elevó a rango de obligatoriedad la paridad de género para el registro de representantes a los distintos cargos de elección popular. Lo anterior obliga a los partidos políticos a incrementar la participación de las mujeres en las contiendas electorales. Sin embargo, únicamente cuatro partidos políticos tuvieron $50 \%$ de representantes femeninos, lo que representa sólo 30\% de los partidos registrados. El resto se ubica en alrededor de 48\% en paridad de género a favor las mujeres (Padilla Loredo y Quintero Soto).

En la LXIII Legislatura de la Cámara de Senadores, las mujeres ocupan únicamente 34\% de los curules², a pesar de su amplia participación en contiendas electorales, lo que no se refleja en su elección como representantes o para desempeñar puestos públicos de decisión. En los estados, la participación de la mujer en las esferas del poder político es aún más raquítica: en la historia de México únicamente seis mujeres han desempeñado el cargo de gobernador constitucional de un estado (de ellas una fue suplente). Los estados con dicha distinción son: Zacatecas, Colima, Tlaxcala, Yucatán y Estado de México. Por lo tanto, de 32 entidades federativas, 27 nunca han sido gobernadas por una mujer.

La inequidad es real, subsiste en el contexto organizacional entre hombres y mujeres, favoreciendo al hombre y señalando a la mujer con una tasa menor de empleoy/o desarrollo profesional. Esto se relaciona con el concepto de techo de cristal, el cual se fundamenta en prejuicios hacia la mujer, que obstaculizan su desarrollo profesional y/o ascenso a posiciones de alto nivel en algunas organizacionales (Agut 
Nieto y Martín Hernández, 2007). Burin (2008) define el concepto techo de cristaß como superficie superior invisible que evita el ascenso en la carrera laboral de una mujer. No obstante, aún cuando se ha descrito y visualizado el problema y se ha logrado implementar cambios en las legislaciones, poco se ha hecho para alcanzar una transformación real a nivel operativo (Burin, 2008).

\section{Las mujeres en el contexto laboral mexicano}

En México persisten estereotipos en torno a la mujer, fundamentados en una cultura machista ${ }^{4}$, que se desprende de generaciones previas y trasciende desde el inconsciente de hombres y mujeres, perpetuando patrones y reproduciendo conductas en torno a hombres y mujeres, permeando en -además de estereotipos culturales - políticas, procedimientos, estructuras, relaciones de poder, creencias, etcétera, de las organizaciones, lo que dificulta el acceso de la mujer a determinados puestos de trabajo.

Imagen 2. Aún cuando han incursionado en campos como las tecnologías de la información, las mujeres no acceden a puestos directivos en dichas áreas. Fuente: fotografía propia.

${ }^{3}$ Es acerca de mujeres con extensa formación profesional, altas calificaciones en sus

trabajos y experiencia, que, sin embargo, en determinado momento su acenso en la organización laboral se ve detenido de forma inexplicable.

${ }^{4}$ El machismo surge de las creencias sociales trasmitidas generacionalmente y perpetuadas dentro de las familias y/u organizaciones sociales, así como en escuelas, grupos sociales y contextos laborales. Consiste, principalmente, en una ideología en donde la mujer es considerada inferior respecto al hombre.

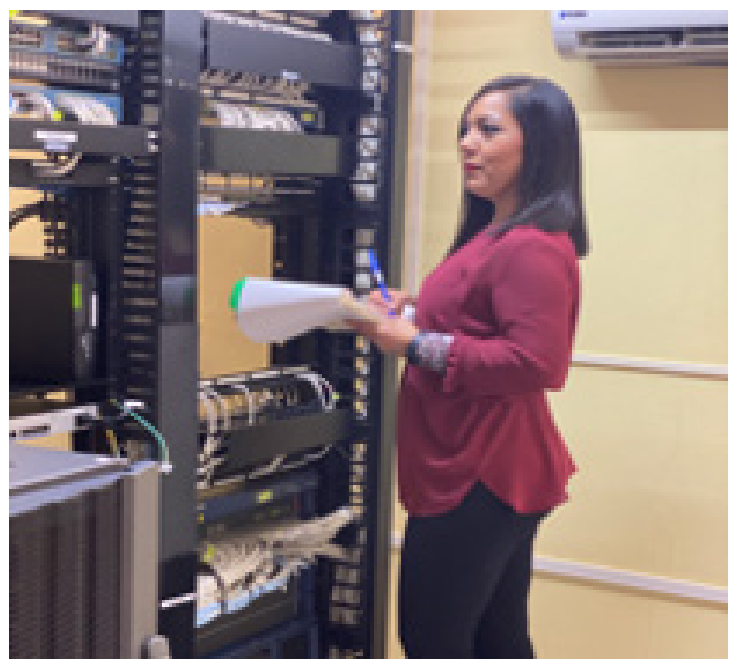

Las Naciones Unidas crearon el programa para el desarrollo, que describe expresamente cómo podrían las democracias emergentes, como la de México, mejorar y ampliar las oportunidades para los seres humanos, sin distinción de género. Crearon el índice específico de medición de la potenciación de género (IPG), que permite medir las oportunidades que se presentan a las mujeres en tres esferas de la vida: la participación política, que ya se mencionó es muy pobre en México y donde predomina la manipulación de las cifras; la participación económica, donde la mujeres, a pesar de ser una fuerza laboral creciente y competente, no alcanzan puestos directivos ni en el sector privado ni público; y el nivel de recursos económicos, en donde es evidente la disparidad salarial acorde con el trabajo desempeñado por hombres o mujeres (Zabludovsky, 2015). Los resultados del IPG muestran la necesidad de continuar hablando y luchando por la equidad de géneros y el empoderamiento social y laboral de la mujer, desde el hogar, la escuela y el ámbito laboral, como un factor importante para el desarrollo del país. 
Imagen 3. Debido al contexto en el que se vive, la maternidad podría provocar dilemas emocionales en las mujeres, referentes a continuar en la escalada del éxito profesional o inclinarse a cuidar de la familia. Fuente: fotografía propia.

${ }^{5}$ La cultura organizacional se refiere a la forma habitual y tradicional de ser de una empresa, compartida en diferentes grados por todos sus miembros y que se aprende y se acepta por los empleados nuevos para ser aceptados como parte de la empresa.
¿El trabajo de las mujeres se considera de menor valía? Estadísticas muestran que las mujeres ocupan actualmente más de $40 \%$ de los puestos laborales del país y participan principalmente en áreas de comercio y servicios donde alcanzan 45.9\% y 44.7\%, respectivamente. Persisten áreas con predominio masculino, en las que la participación de la mujer se ha visto considerablemente restringida, por ejemplo: el poder judicial, la suprema corte y la construcción. Adicionalmente, las percepciones económicas de los empleados parecen estar vinculadas con su género: mientras que el porcentaje de hombres que percibe salario mínimo es de $5.1 \%$, las mujeres pueden llegar hasta 13.4\%. En el otro extremo, el porcentaje de hombres que llega a percibir más de cinco salarios mínimos es de 17.4\%, comparado con el $10.7 \%$ de las mujeres. Lo anterior genera dudas en torno a la existencia de la equidad de género (Zabludovsky, 2007).

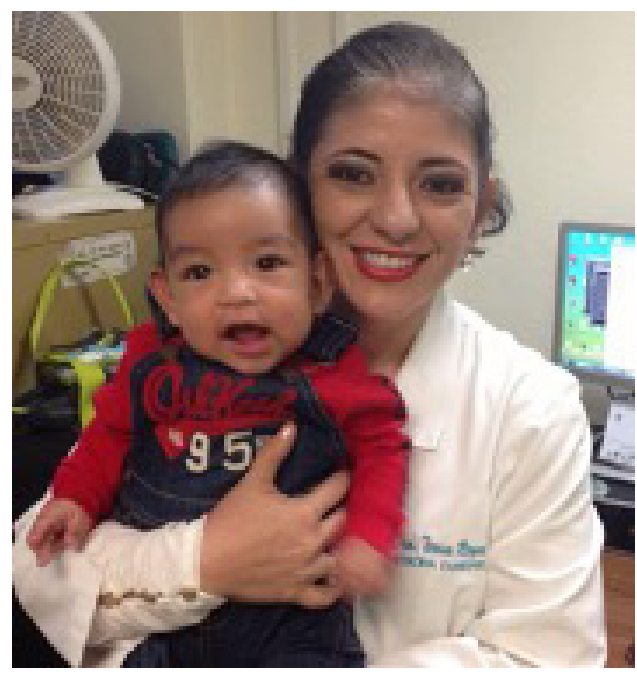

Así, el poder de la mujer se ha quedado en el discurso público, ya que persiste inequidad en oportunidades de desarrollo en el ámbito organizacional. Esto se manifiesta en la ausencia de mujeres en cargos directivos, tanto en organizaciones públicas como privadas, consecuencia de su cultura organizacional ${ }^{5}$. Ésta implica la existencia de patrones culturales al interior de la organización, que tienen como consecuencia percepciones sobre diferencias entre los géneros, que a su vez reproducen y perpetúan significados y jerarquías estereotipadas, sobre las que se fundamentan relaciones asimétricas de poder. Pareciera que las oportunidades para hombres y mujeres son diferentes y los perfiles laborales también, así, un hombre con menor experiencia y menor grado escolar tiene mayores probabilidades de ser ascendido en la empresa que una mujer, aun cuando ella cuente con mayores habilidades, conocimientos y destrezas que su contraparte masculina. ¿Por qué se genera esto?

Los patrones culturales arraigados en el consciente y subconsciente de los individuos de una organización se filtran a la cultura organizacional, que en el contexto mexicano se refiere al machismo dentro y fuera de la empresa (Cerva Cerna, 2014). Es así como entre los principales obstáculos que una mujer podría enfrentar para ascender en la unidad de mando estarían las dificultades para compaginar las diferentes áreas de vida —en el sentido de lo que se espera de ellas —con las demandas propias de las actividades laborales. Esto implicaría que la mujer aceptara su rol dentro de la organización como principal y se involucrara totalmente en su desarrollo profesional, lo que generaría un dilema emocional difícil de resolver, que sería el cómo conjugar ambos roles, lo que podría desembocar en la elección del rol de madre sobre el rol de profesional exitosa. Otras limitantes serían las políticas internas de las organizaciones en relación con las mujeres: los empleadores suelen argumentar que el estado 
incierto de maternidad y su consecuente limitación laboral por "incapacidad" hace que las mujeres vean limitadas las oportunidades de capacitación, ascenso o permanencia dentro de la organización (Villegas y Castañeda, 2017).

\section{¿A quién culpar por la desventura de las mujeres?}

Las mujeres, en ocasiones, renuncian a escalar en la pirámide organizacional debido a un conjunto de factores sociales, psicológicos y culturales. Lamentablemente, esta situación se puede volver un círculo vicioso en el que se perpetúan los patrones de sumisión.

Con el objetivo de continuar con su desarrollo profesional, pero en el contexto de la sociedad mexicana, algunas mujeres optan por esquemas profesionales que típicamente han sido asociados con el género, como docentes, enfermeras, asistentes, etcétera. Otras enmarcan su futuro profesional a trabajos informales autodirigidos desde casa o asistenciales debido a que la sociedad patriarcal les ha atribuido (atención del hogar, cuidado de los hijos, cesión de sus derechos en favor de los intereses del marido, presiones sociales, etcétera). En este sentido, las mujeres también se encuentran afectadas por patrones que se han establecido y que han perdurado en la cultura de una sociedad y que se filtran hasta las organizaciones.

Imagen 4. Las mujeres han logrado permanecer y desarrollarse dentro del campo de la salud, sin embargo, los puestos administrativos de alta dirección aún pertenecen a los hombres. Fuente: fotografía propia.

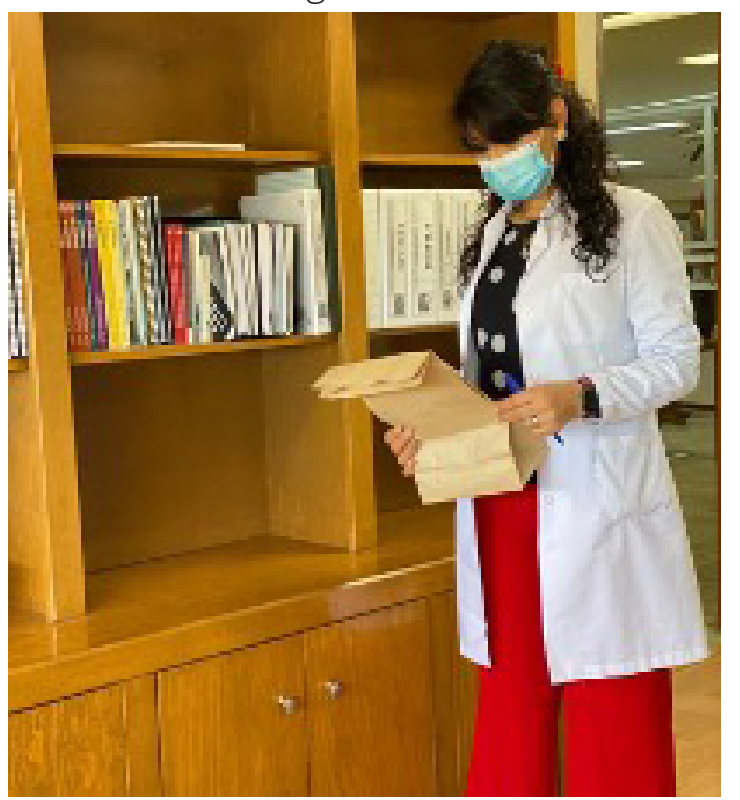

En México, la tasa bruta de nupcialidad es de 4.6 matrimonios por cada mil habitantes para el año 2015, lo que implica una disminución de 34.3\% con respecto al año 2000 (INEGI, 2015). Esto podría traducirse en que el estado de matrimonio ha dejado de ser parte importante del desarrollo personal, pues dicha tasa implica una disminución de 34.3\% con respecto al año 2000 (INEGI, 2015). Por ello, es importante ir cambiando actitudes, concepciones y prácticas culturales. 
Hablar sobre diferencias de género en las organizaciones parece ser un tema ya rebasado, una situación del pasado. Sin embargo, sigue siendo una manifestación de poder masculino lo que impera al interior de las organizaciones. Es evidente que los espacios y áreas para el desarrollo de las mujeres permanecen situadas en "áreas femeninas", como lo social (recursos humanos, actividades de cultura, en el cuidado de otros, dentro de organizaciones y luchas sociales). Al interior de las organizaciones, la estructura permanece rígida, la cultura organizacional no ha cambiado en referencia al posicionamiento de cada género. En este sentido, persiste la necesidad de un cambio estructural profundo dentro de las organizaciones, que implique la transformación de la cultura organizacional donde la regla sea la inclusión.

\section{Una lucha de género}

A criterio personal, los hombres y las mujeres son y serán diferentes siempre, por lo tanto, es indispensable reconocer los aportes y el valor que cada género tiene, trabajar en colaboración como equipo, con un fin común. Es a través del ejercicio del respeto, la comunicación y el reconocimiento del valor del otro que se logrará acceder a mayores éxitos dentro de la vida laboral, social y familiar, y que la equidad se convertirá en un elemento de la cultura universal.

Corresponde a hombres y mujeres establecer e implementar las condiciones de este camino de cambio, así como las formas que hagan realidad los discursos políticos, promesas y planteamientos añejos. Hay que enfocarse en las trasformaciones culturales necesarias que permitan hacer realidad los cambios.

Es tiempo de romper estereotipos para modificar conductas, dejar de ser parte del problema para contribuir en la solución, a partir de hombres y mujeres comprometidos con la equidad y el respeto mutuo, que sean modelos a seguir para las generaciones de hoy y las del mañana, donde la constante sean los hechos y no las promesas.

\section{Referencias}

* Agut Nieto, S. y Martín Hernández, P. (2007). Factores que dificultan el acceso de las mujeres a puestos de responsabilidad: una revisión teórica. Apuntes de psicología, 25(2), 201-214. http://www.apuntesdepsicologia.es/index.php/revista/ article/view/125

* Bernal González, M. del C., Moreno Maya, A. y Equipo directivo del cimad. (s.f.). ¿Por qué hacer visible el talento femenino? http://itandlifestyle.com/por-que-hacervisible-el-talento-femenino/

* Burin, M. (2008). Las "fronteras de cristal" en la carrera laboral de las mujeres. Género, subjetividad y globalización. Anuario de Psicología, 39(1), 75-86. https:// www.redalyc.org/articulo.oa?id=97017401006 
* Cerva Cerna, D. (2014). Participación política y violencia de género en México. Revista mexicana de ciencias políticas y sociales, 59(222), 117-140. http://www.scielo. org.mx/scielo.php?script=sci_arttext\&pid=S0185-19182014000300005\&lng=es\& tlng=es

* Deloitte y Alliace for Board Diversity. (2019). The missing pieces report: the 2018 Board Diversity Census of women and minorities on Fortune 500 boards. https://cutt. $\mathrm{ly} / \mathrm{n} \times \mathrm{ZV} \mathrm{VXX}$

* Instituto Nacional de Estadística y Geografía (INEGI). (2015). Panorama sociodemográfico de México 2015. http://seieg.iplaneg.net/seieg/doc/Panorama_ Sociodemografico_2015_1452886126.pdf

- Martinez Villegas, J. A. y Camacho Castañeda, C. L. (2017). Estereotipo, prejuicio y discriminación hacia las mujeres en el contexto laboral latinoamericano. Cuestiones de género: de la igualdad y la diferencia, (12), 347-364. http://dx.doi.org/10.18002/ cg.v0i12.4409

* Padilla Loredo, S., y Quintero Soto, M. L. Mujer y democracia. Paridad de género en la política mexicana. https://cutt.ly/DxzVSy3

* Zabludovsky, G. (2007). Las mujeres en México: trabajo, educación superior y esferas de poder. Política y Cultura, (28), 9-41. https://www.redalyc.org/articulo. oa?id=26702802

- Zabludovsky, G. (2015). Las mujeres en los ámbitos de poder económico y político de México. Revista Mexicana de Ciencias Políticas y Sociales, 60(223), 61-94. http:// dx.doi.org/10.1016/S0185-1918(15)72131-8

\section{Cómo CITAR ESTE ARTículo}

* Reyes Chacon, María Teresa. (2021, mayo-junio). ¿En dónde quedó el poder de las mujeres? La problemática de la brecha de género. Revista Digital Universitaria (RDU), 22(3). Dol: http://doi.org/10.22201/cuaieed.16076079e.2021.22.3.1. 